\title{
Activating Unconventional Wavelength Bands for Coherent Optical Communication by On-chip Continuous Spectral Translation
}

Deming Kong ( $\nabla$ dmkon@fotonik.dtu.dk)

Technical University of Denmark https://orcid.org/0000-0001-6552-4081

Yong Liu

Technical University of Denmark

Zhengqi Ren

University of Southampton

Yongmin Jung

University of Southampton

Chanju Kim

Technical University of Denmark https://orcid.org/0000-0001-8011-1904

\section{Yong Chen}

University of Southampton

Natalie V. Wheeler

University of Southampton

Marco N. Petrovich

University of Southampton

Minhao Pu

Technical University of Denmark https://orcid.org/0000-0002-4993-4299

Kresten Yvind

Technical University of Denmark https://orcid.org/0000-0001-8013-1606

Michael Galili

Technical University of Denmark

Leif Oxenløwe

Technical University of Denmark https://orcid.org/0000-0001-6013-3639

David Richardson

University of Southampton

$\mathrm{HaO} \mathrm{Hu}$

Technical University of Denmark https://orcid.org/0000-0002-8859-0986 


\section{Keywords:}

Posted Date: January 20th, 2022

DOI: https://doi.org/10.21203/rs.3.rs-1086400/v1

License: (c) (1) This work is licensed under a Creative Commons Attribution 4.0 International License. Read Full License

Version of Record: A version of this preprint was published at Nature Communications on July 16th, 2022. See the published version at https://doi.org/10.1038/s41467-022-31884-2. 


\title{
Activating Unconventional Wavelength Bands
} for Coherent Optical Communication

\section{by On-chip Continuous Spectral Translation}

\author{
Deming Kong ${ }^{1, *}$, Yong Liu ${ }^{1}$, Zhengqi Ren², Yongmin Jung ${ }^{2}$, Chanju Kim¹, Yong Chen², \\ Natalie V Wheeler ${ }^{2}$, Marco N Petrovich², Minhao $\mathrm{Pu}^{1}$, Kresten Yvind ${ }^{1}$, Michael Galili ${ }^{1}$, \\ Leif K Oxenløwe ${ }^{1}$, David J Richardson ${ }^{2}$, and $\mathrm{Hao} \mathrm{Hu}^{1, *}$ \\ ${ }^{1}$ DTU Fotonik, Technical University of Denmark, DK-2800, Kgs. Lyngby, Denmark \\ ${ }^{2}$ Optoelectronics Research Centre, University of Southampton, Southampton, SO17 1BJ, UK
}

\section{Contributions}

D.K. and H.H conceived the concept and the experiment; Y.L. designed the AlGaAsOI nanowaveguides, supervised by H.H. and M.P; Y.L. and D.K. performed the simulation of the AlGaAsOI nanowaveguides; Y.L., C.K., and K.Y. fabricated the AlGaAsOI nanowaveguides; D.K. and Y.L. characterized the AlGaAsOI nanowaveguides, and H.H. and M.P provided suggestions; Z.R. and Y.J. designed and fabricated the 1.74$\mu \mathrm{m}$ TDFA, supervised by D.J.R.; M.N.P, Y.C. and N.W. designed and fabricated the 19-cell HCF, supervised by D.J.R.; D.K. constructed the experiment setup, performed the experiment and processed the data; H.H. supervised the experiment; D.K., H.H., Y.L., Y.J., M.P, M.G., L.K.O., and D.J.R. discussed the results; The manuscript was written by D.K., Y.J., and H.H., and all authors contributed to the writing; H.H., L.K.O., and D.J.R. supervised the projects.

\section{Corresponding authors}

Correspondence to: Deming Kong (dmkon@fotonik.dtu.dk), ORCID: 0000-0001-6552-4081

Hao Hu (huhao@fotonik.dtu.dk), ORCID: 0000-0002-8859-0986 


\section{Abstract}

Despite being perceived historically to provide "unlimited" bandwidth, today's optical communication systems are fast approaching their capacity limits in the conventional telecom bands ${ }^{1}$. To satisfy the everincreasing capacity demand, opening up new wavelength bands is becoming an appealing solution both in cabled and free-space optical communications in the transparent windows ${ }^{2}$. However, this would ordinarily require the development of whole optical transceivers for any new wavelength band, which is both timeconsuming and expensive. Here, we present an on-chip continuous spectral translation based coherent optical communication approach that can exploit existing commercial transceivers to unlock the vast and currently unused potential new wavelength bands rather than dictate the need to develop new coherent transceivers operating directly in those bands. The spectral translators are continuous-wave pumped aluminium gallium arsenide on insulator (AlGaAsOI) nanowaveguides that can provide a continuous conversion bandwidth over an octave. This enables unprecedented coherent transmission in the $2-\mu \mathrm{m}$ wavelength band using well-developed conventional C-band transmitters and coherent receivers. We demonstrate $318.25-\mathrm{Gbit} \mathrm{s}^{-1}$ Nyquist wavelength-division multiplexed coherent transmission over a 1.15-km hollow-core fibre in the 2$\mu \mathrm{m}$ wavelength band using this approach. Our demonstration paves the way for coherently transmitting, detecting, and processing signals at wavelength bands beyond the capability of today's devices.

\section{Main}

Coherent optical communication is a truly revolutionary technology that has transformed old and overburdened optical fibre networks into data superhighways. Owing to its overwhelming advantages in terms of spectral efficiency and receiver sensitivity ${ }^{3}$, coherent optical communication has significantly increased the capacity and transmission distance of fibre networks and has been critical to meeting the ever-increasing data traffic demands of the worldwide internet. Today, coherent technology has expanded its applications from long-haul, large-capacity transmission to metro networks and short-reach links ${ }^{4}$ due to advances in electronic and photonic devices which have enabled tremendous cost and power consumption reductions ${ }^{5}$. Using coherent optical communication, information can be encoded onto optical carriers with advanced modulation $^{6,7}$ and multiplexing ${ }^{8-10}$ in the complex domain by I/Q modulators. The complex field of the signal can be completely recovered after transmission by a coherent receiver, in which the linear transmis- 
sion impairments such as chromatic dispersion can be fully compensated by digital signal processing ${ }^{11,12}$. Yet, coherent transceivers need to be specially designed and are only available for the conventional $\mathrm{C}$ and $\mathrm{L}$ bands ${ }^{13,14}$, as well as recently the $\mathrm{S}$ band ${ }^{15}$. Today, coherent optical communication systems in the conventional wavelength bands are reaching their capacity limits ${ }^{1,16,17}$. The potential to open up new wavelength bands is now attracting significant interest ${ }^{18}$. New fibre technologies have emerged which give rise to the possibility of transmitting optical signals for instance in the $2-\mu \mathrm{m}_{\text {band }}{ }^{19-22}$ and the $1-\mu \mathrm{m}$ band ${ }^{23-25}$. Also, an ambitious vision of utilizing the huge bandwidth resources of free space could support a possible universal solution for future ubiquitous optical communications with unlimited sustainability ${ }^{2}$. However, it is very challenging to build up a complete coherent optical communication system for unconventional wavelength bands due to the lack of narrow-linewidth lasers, I/Q modulators, and coherent receivers (including $90^{\circ}$ optical hybrids and balanced photodetectors). Developing these crucial devices is a cost-intensive and time-consuming process and it has been a major obstacle to opening up new wavelength bands. Currently, coherent optical communication has not yet been demonstrated in any unconventional wavelength band.

An alternative solution is to use spectral translation technology to provide a bridge between mature telecom bands and unconventional wavelength bands. By translating the wavelength of the signal forth and back using degenerate four-wave mixing (FWM), one can effectively build coherent transmitters and receivers for unconventional bands. Demonstrations based on silicon nanowaveguides have shown the feasibility of bridging the telecom bands and the $2-\mu \mathrm{m}$ band ${ }^{26,27}$, exhibiting a large conversion bandwidth of $820 \mathrm{~nm}^{26}$. However, the conversion efficiency is limited due to intrinsic material properties and two-photon absorption (TPA) induced nonlinear loss. Thus, picosecond pulsed lasers with a high peak power are required for the pump. The need for a pulsed pump adds complexity and severely limits the speed and spectral efficiency of the signal. Recently, a highly efficient 650 -nm spectral translation based on a continuous-wave (CW) pump has been demonstrated using a silicon nitride $\left(\mathrm{Si}_{3} \mathrm{~N}_{4}\right)$ microring resonator ${ }^{28}$. However, the nature of the resonance gives a discrete translation band in frequency and narrow resonance bandwidth, which significantly limits the data rate and compromises efficient bandwidth usage within these unconventional bands. Aiming for coherent optical communication with high speed and high spectral efficiency in unconventional wavelength bands, continuous (in both time and frequency) spectral translation is necessary but has not yet been demonstrated.

Here, we propose and demonstrate continuous spectral translation for coherent optical communication in unconventional wavelength bands, avoiding the need to develop coherent optical transceivers in those 


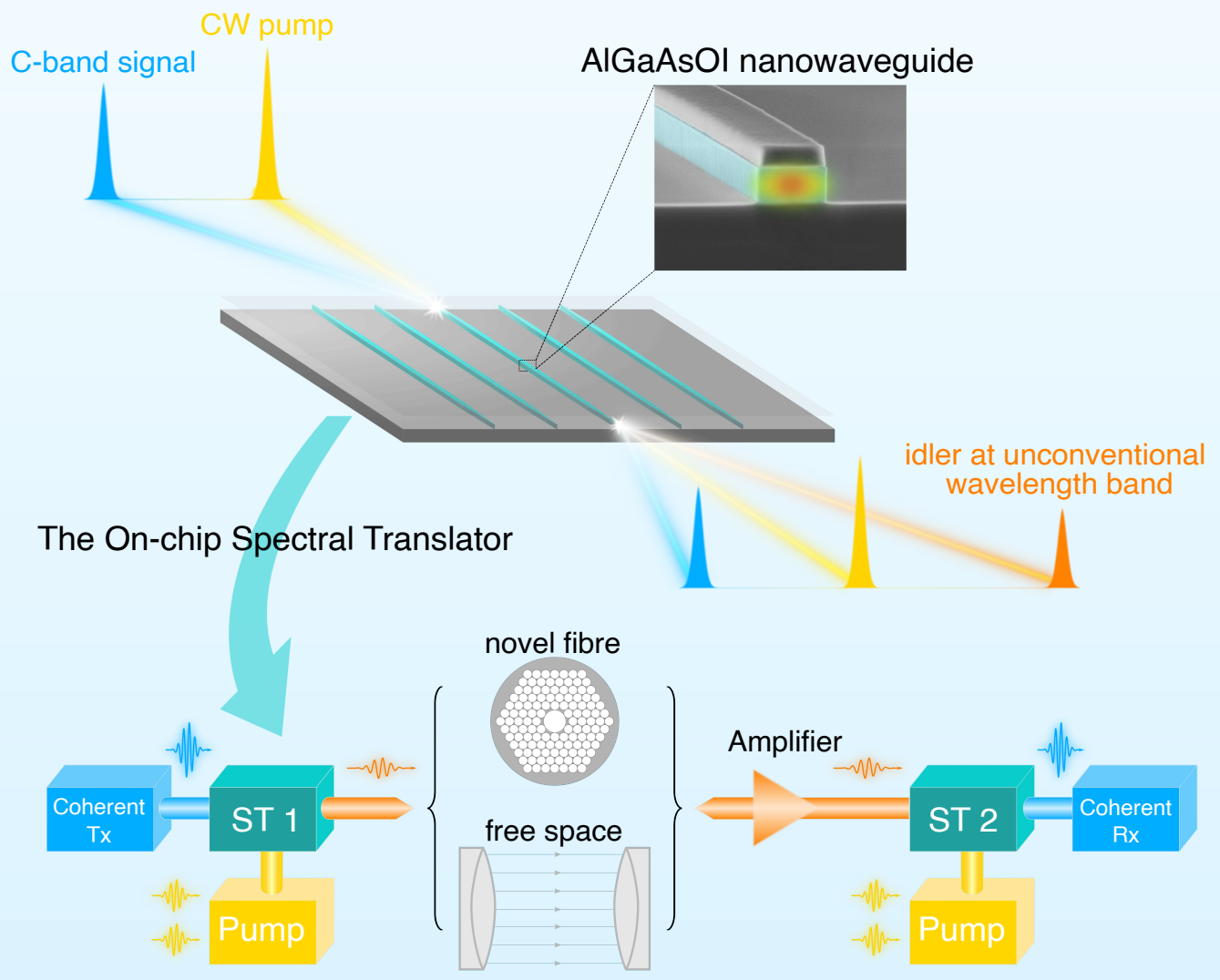

Coherent optical communication at unconventional wavelength bands

Fig. 1 | Coherent optical communication at unconventional wavelength bands enabled by on-chip continuous wave spectral translation. The spectral translators are based on CW-pumped FWM in AlGaAsOI nanowaveguides where a continuous conversion bandwidth over an octave can be achieved with reasonable conversion efficiency. Using a pair of spectral translators (ST1 and ST2), an optical coherent transmitter and a receiver working at new wavelength bands can be realized with high-performance C-band counterparts. Such a spectral translator based coherent optical communication scheme has the potential to utilize the vast unexploited wavelength bands possible in novel fibres or free space without the need to develop optical transceivers at those wavelength bands. 
bands. As shown in Fig. 1, the basic principle is to bridge between the mature telecom $1.55-\mu \mathrm{m}$ band $(\mathrm{C}$ band) and the new band by spectrally translating the signal forth and back using an on-chip solution based on CW-pumped degenerate FWM in AlGaAsOI nanowaveguides. We can then effectively build a pair of optical coherent transmitters and receivers that are compatible with advanced modulation and multiplexing technologies working in the new wavelength band based on mature C-band devices. At the transmitter side, advanced modulation and optical multiplexing such as quadrature-amplitude modulation (QAM) and Nyquist wavelength-division multiplexing (N-WDM) can be utilized and the high spectral efficiency signals converted to the new wavelength band. At the receiver side where the signal is converted back to the $\mathrm{C}$ band, coherent detection with advanced digital signal processing can be enabled for high performance and tolerance to linear impairments from the transceiver and transmission.

To achieve continuous spectral translation, both $\mathrm{CW}$ pumping and a non-resonant structure are needed, which puts a very strict requirement on the integrated nonlinear platform. Our work is based on recent advances in ultra-efficient AlGaAsOI nonlinear nanowaveguides ${ }^{29}$. The high refractive index contrast between the AlGaAs and the silica insulator can strongly confine light in the waveguide, enabling a very high conversion efficiency (CE) and bandwidth product amongst the various nonlinear material platforms ${ }^{30}$. The AlGaAsOI nanowaveguide can also be dispersion engineered to accommodate high-order phase matching for a very large conversion bandwidth of over an octave $\mathrm{e}^{31,32}$, supporting spectral translation with flexible target wavelength bands. The energy bandgap of AlGaAs can also be engineered by tuning the aluminium concentration, so that the nanowaveguide can be made free from TPA when pumping at the desired wavelength band, increasing the $\mathrm{CE}$. These unique features make the AlGaAsOI nanowaveguide an appealing solution for continuous spectral translation.

Here, continuous spectral translation between the $\mathrm{C}$ band and the $2-\mu \mathrm{m}$ band is explored and demonstrated, since the $2-\mu \mathrm{m}$ band is becoming a leading contender for optical communication amongst new wavelength bands (Supplementary Information). To the best of our knowledge, optical communication in the $2-\mu \mathrm{m}$ band has only been focused on intensity-modulation and direct-detection systems ${ }^{19-22,33}$. To build a $2-\mu \mathrm{m}-$ band coherent optical communication system based on spectral translation, we need a sufficiently powerful 1.74- $\mu \mathrm{m}$ pump and AlGaAsOI nanowaveguides with a conversion bandwidth $>450 \mathrm{~nm}$. For the $1.74-\mu \mathrm{m}$ pump, we have developed an all-fibre short-wavelength thulium-doped fibre amplifier (TDFA) working in the 1700-1800 $\mathrm{nm}$ band, achieving an output power of $>500 \mathrm{~mW}^{34}$. The design and fabrication details of the short-wavelength TDFA are given in the Methods. 

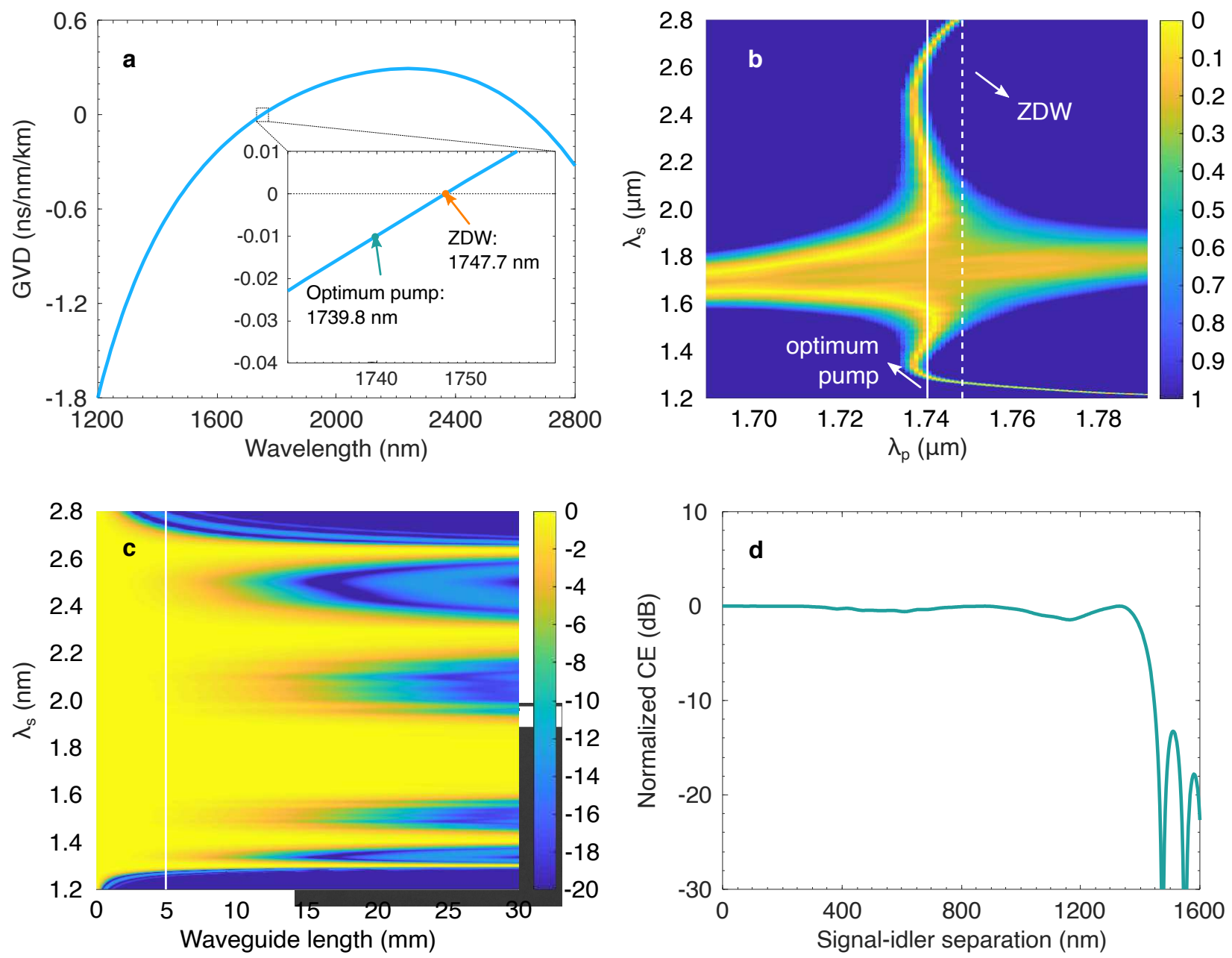

Fig. 2 Simulation results of the AIGaAsOI nanowaveguide. a, Dispersion profile of the AlGaAsOI nanowaveguide. The optimum pump wavelength $(1739.8 \mathrm{~nm})$ is shifted from the ZDW $(1747.7 \mathrm{~nm})$ to utilize the high-order phase matching, where the fundamental conversion band can be merged with the highorder phase matching band for an extended conversion bandwidth. b, Normalised phase mismatch $|\Delta \beta L / \pi|$ as a function of pump and signal wavelengths, with a waveguide length of $5 \mathrm{~mm}$. The white straight solid and dash-dotted lines indicate the optimum pump wavelength and the ZDW. c, Normalised CW as a function of waveguide length and signal wavelength with the pump at the optimum wavelength. d, Normalised CE versus signal-idler separation with a 5-mm-long AlGaAsOI nanowaveguide, when the pump is located at the optimum wavelength. The simulation results show a conversion bandwidth of over $1400 \mathrm{~nm}$. 
We have first explored the conversion bandwidth of the AlGaAsOI nanowaveguide using a numerical simulation. The geometric dimensions of the AlGaAsOI nanowaveguides are $910 \mathrm{~nm}$ in width, $350 \mathrm{~nm}$ in height, and $5 \mathrm{~mm}$ in length. Figure 2a shows the simulation results of the dispersion profile of the AlGaAsOI nanowaveguide. The zero-dispersion wavelength (ZDW) is around $1747.7 \mathrm{~nm}$. High-order phase matching can be used to expand the conversion bandwidth by combining both the fundamental phase matching band and high-order phase matching bands, albeit at some expense in $\mathrm{CE}^{31,32}$ (Supplementary Information). Figure $2 \mathrm{~b}$ illustrates the normalised phase mismatch $|\Delta \beta L / \pi|$ ( $\Delta \beta$ is the phase mismatch and $L$ is the length of the waveguide, see Supplementary Information) as a function of pump and signal wavelengths, for a waveguide length of $5 \mathrm{~mm}$. We see that a much wider continuous conversion bandwidth can be achieved by exploiting the fundamental and high-order phase matching bands, by slightly detuning the pump from the ZDW to the optimum wavelength at $1739.8 \mathrm{~nm}$. Figure $2 \mathrm{c}$ shows the normalised CE (defined to characterize the wavelength-dependent conversion efficiency, see Supplementary Information) on a decibel scale against the length of the nanowaveguide and the signal wavelength when the pump is set to optimum wavelength. The fundamental and high-order phase matching bands start to split when the length of the nanowaveguide increases. A synergetic conversion band is lost when the length surpasses the designed $5 \mathrm{~mm}$. The conversion enables the continuous spectral translation from $1285.2 \mathrm{~nm}(233.27 \mathrm{THz})$ to $2691.9 \mathrm{~nm}(111.37 \mathrm{THz})$, spanning an octave. Figure $2 \mathrm{~d}$ gives the normalised $\mathrm{CE}$ against the signal-idler separation in nanometres when the length of the nanowaveguide is set to $5 \mathrm{~mm}$. Note that the conversion bandwidth is defined as the 3-dB width of the normalised CE against signal-idler separation. The overall conversion bandwidth is larger than $1400 \mathrm{~nm}$ with a flat (within $1 \mathrm{~dB}$ variation) conversion bandwidth over $1000 \mathrm{~nm}$. The fabrication details are given in the Methods.

The continuous spectral translation is tested within the scenario of multidimensional modulation and multiplexing, in particular 32 Gbaud signal with 16-QAM format and four N-WDM channels. Figure 3a shows the experimental setup. At the transmitter side, the $4 \times 32$ Gbaud 16-QAM signal is firstly generated using mature C-band external cavity lasers and I/Q modulators with a spacing of $33 \mathrm{GHz}$ between adjacent channels. The pump originates from a CW distributed feedback (DFB) laser thermally tuned to a central wavelength of $1739.74 \mathrm{~nm}$ with a linewidth of $2 \mathrm{MHz}$. To achieve a sufficient CE, the CW pump is amplified by the in-house built short-wavelength TDFA and split into two tributaries, with measured optical powers of $20.0 \mathrm{dBm}$ and $19.9 \mathrm{dBm}$ before the input tapered couplers of $\mathrm{AlGaAsOI}$ nanowaveguides 1 and 2 , respectively. The transmitter-side spectral translator converts the N-WDM signal to the $2-\mu \mathrm{m}$ band. A 
a
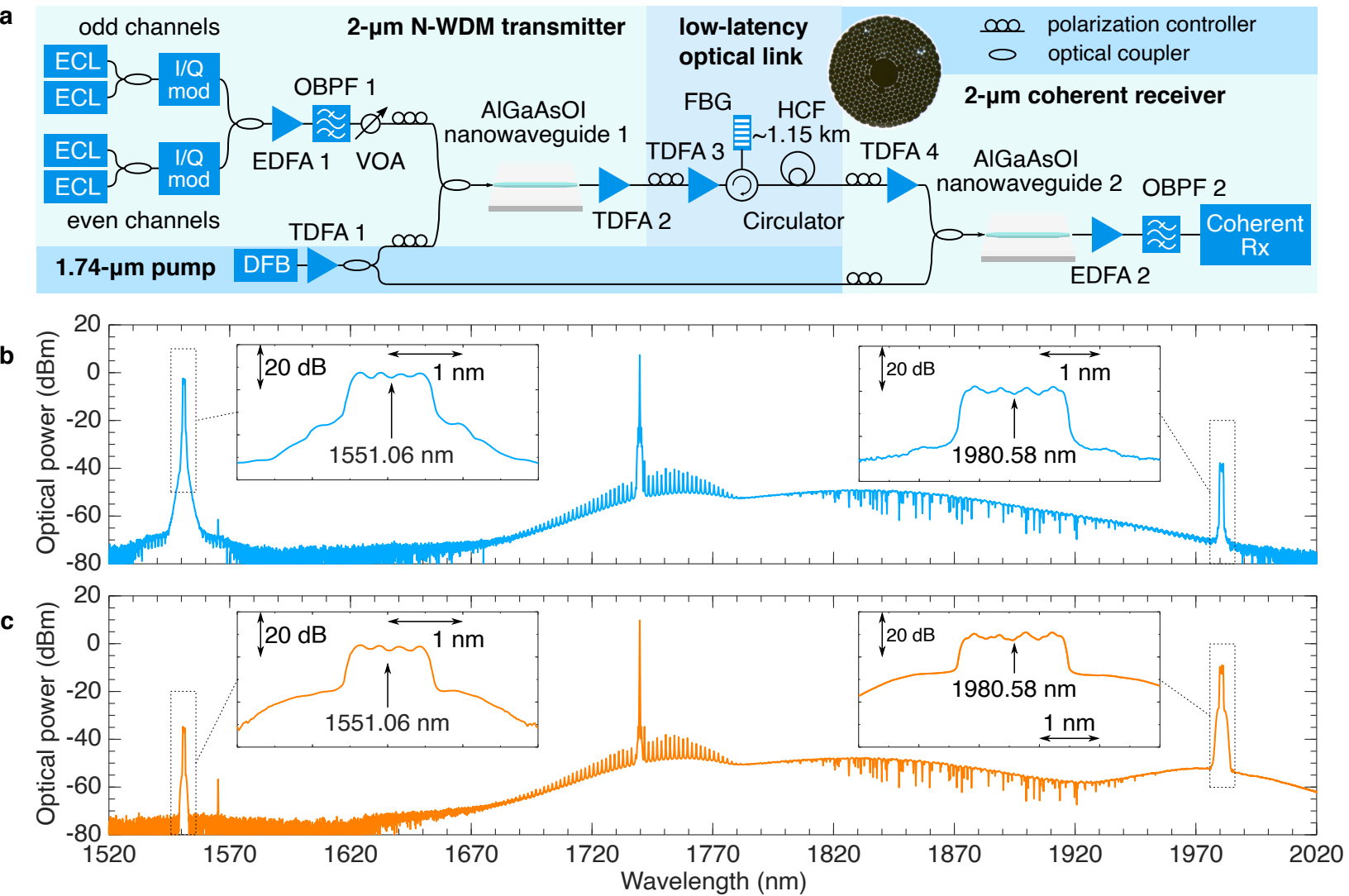

Fig. 3 | 2- $\mu$ m-band coherent transmission based on a pair of spectral translators with $4 \times 32$ Gbaud

16-QAM signal. a, The $2-\mu \mathrm{m}-\mathrm{band} \mathrm{N}-\mathrm{WDM}$ transmitter is based on a spectral translator (AlGaAsOI nanowaveguide 1) to convert the C-band signal to the $2-\mu \mathrm{m}$ band. The $2-\mu \mathrm{m}$-band coherent receiver is based on a second spectral translator (AlGaAsOI nanowaveguide 2) to convert the 2- $\mu \mathrm{m}$-band signal back into the $\mathrm{C}$ band. The pump for the spectral translators is generated through a DFB laser working at $1739.74 \mathrm{~nm}$ and an in-house designed short-wavelength TDFA (TDFA 1). The 2- $\mu$ m-band signal is transmitted through a $\sim 1.15-\mathrm{km}$ HCF link with a low latency of $3.82 \mu \mathrm{s}$. Inset shows a cross-sectional image of the 19-cell HCF. b, Transmitter-side spectral translation from $1551.06 \mathrm{~nm}$ to $1980.58 \mathrm{~nm}$ with an output CE of $-35.4 \mathrm{~dB}$. c, Receiver-side spectral translation from $1980.58 \mathrm{~nm}$ back to $1551.06 \mathrm{~nm}$ with an output $\mathrm{CE}$ of $-24.5 \mathrm{~dB}$. The difference in the $\mathrm{CE}$ is due to the difference in the AlGaAsOI nanowaveguides and the fact that the 2- $\mu \mathrm{m}$-band signal experiences more loss during coupling and propagation. 
2- $\mu \mathrm{m}$-band TDFA is used to amplify the translated N-WDM signal and reject the residual C-band signal and the pump.

We transmitted the 2- $\mu \mathrm{m}$-band $4 \times 32$-Gbaud 16-QAM signal over a $\sim 1.15$-km low-latency hollow-core fibre (hollow-core fibre (HCF), i.e., a 19-cell hollow-core photonic bandgap fibre ${ }^{19}$ (see Methods for further details)). The HCF has a flat-topped transmission window with the 3-dB width extending from $1959 \mathrm{~nm}$ to $2045 \mathrm{~nm}$, with a minimum loss of $2.8 \mathrm{~dB} \mathrm{~km}^{-1}$. It is spliced to two standard single-mode fibre (SSMF) patch cords, giving an overall measured loss of $9 \mathrm{~dB}$. The extra loss is mostly due to a large mode-field diameter mismatch between the HCF and the SSMF. The measured latency (i.e., pulse propagation delay) of the HCF is $3.82 \mu \mathrm{s}$ (Supplementary Information).

At the receiver side, the $2-\mu \mathrm{m}$-band N-WDM signal is amplified and launched into the receiver-side spectral translator, where the 2- $\mu \mathrm{m}$-band signal is converted back into the $\mathrm{C}$ band. An erbium-doped fibre amplifier (EDFA) amplifies the C-band N-WDM signal and rejects the residual 2- $\mu$ m-band signal and the pump. The N-WDM signal is launched into a C-band coherent receiver for detection and performance evaluation. More details about the experimental setup, including the signal generation, detection, and digital signal processing are described in the Methods.

The optical spectra of the transmitter-side and receiver-side spectral translations are shown in Fig. $3 \mathrm{~b}$ and Fig. 3c, respectively. The spectra are measured at the output of the AlGaAsOI nanowaveguides, thus the coupling losses are included. The transmitter-side spectral translator converts the $4 \times 32$-Gbaud 16 -QAM signal centred at $1551.06 \mathrm{~nm}$ to a centre wavelength at $1980.58 \mathrm{~nm}$. The transmitter-side CE is measured to be $-35.4 \mathrm{~dB}$. The receiver-side spectral translator converts the $2-\mu \mathrm{m}$-band signal back into the C-band with a centre wavelength of $1551.06 \mathrm{~nm}$, with a measured CE of $-24.5 \mathrm{~dB}$. The difference in the CEs is mainly due to the larger coupling loss and waveguide loss experienced by the $2-\mu \mathrm{m}$-band signal than the C-band signal, as well as the difference between the AlGaAsOI nanowaveguides.

We evaluated the 2- $\mu \mathrm{m}$-band transmission system by measuring the bit error ratio (BER) performance of a single 16-QAM channel, in the back-to-back scenario (Fig. 4a) and after 1.15-km HCF transmission (Fig. $4 \mathrm{~b}$ ). The received optical power is measured before the TDFA (TDFA 4 shown in Fig. 3) in the 2- $\mu$ m-band coherent receiver. Since the $1.74-\mu \mathrm{m}$ pump laser has a large linewidth of $2 \mathrm{MHz}$, we have observed severe cycle slips $^{35}$ when processing the received signal. Therefore, we used a $20 \%$ pilot overhead in the carrier recovery to remove the cycle slips. The pilot overhead could be significantly reduced if a carrier recovery algorithm with high tolerance to phase noise is used ${ }^{36,37}$ or a narrow linewidth laser is utilized for the 1.74- 

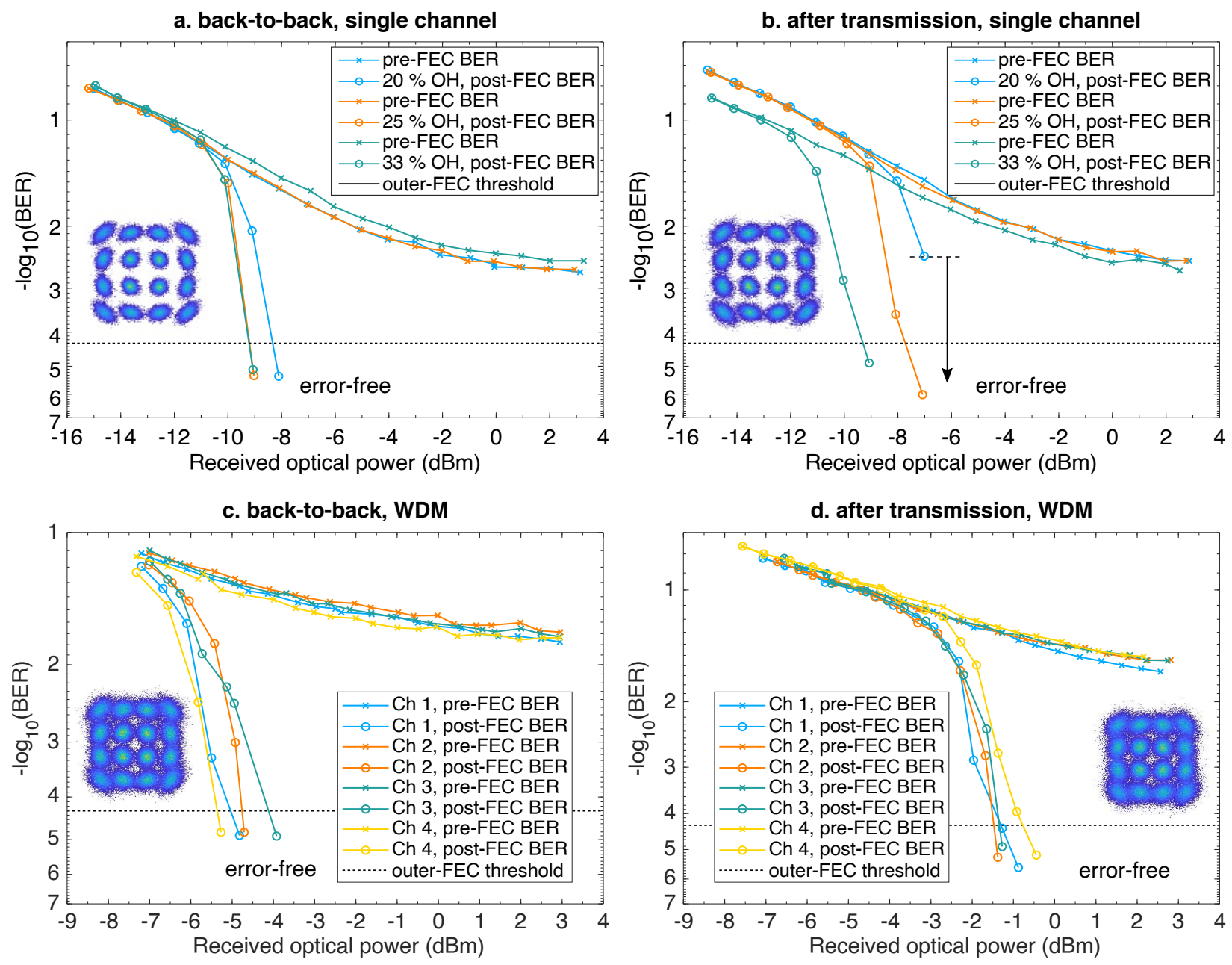

Fig. 4 | BER measurement for the 2- $\mu \mathrm{m}$-band signals using the $2-\mu \mathrm{m}$-band transmitter and receiver based on the spectral translators. a, BER performance of the single-channel 2- $\mu \mathrm{m}$-band 16-QAM signal at back-to-back. b, BER performance of the single-channel 2- $\mu \mathrm{m}$-band 16-QAM signal after 1.15-km HCF transmission. Error-free performance is achieved for both back-to-back and transmission scenarios with $20 \%, 25 \%$, and $33 \%$ LDPC overheads, indicating a good performance margin. c, BER performance of the 2- $\mu \mathrm{m}$-band $4 \times 32$ Gbaud 16-QAM signal at back-to-back. d, BER performance of the $2-\mu \mathrm{m}$-band $4 \times 32$ Gbaud 16-QAM signal after 1.15-km HCF transmission. With 33\% LDPC overhead, error-free performance is achieved for both back-to-back and transmission scenarios. Insets give typical signal eye diagrams for performance references. 
$\mu \mathrm{m}$-band pump $^{38}$. We have investigated the use of low-density parity-check (LDPC) code overheads of $20 \%, 25 \%$, and $33 \%$. We assume a $0.8 \%$ overhead for outer-hard-decision forward error correction (HDFEC) to eliminate the well-known BER floor from the LDPC decoding ${ }^{39}$. We can conclude that all cases result in error-free performance after LDPC decoding. A $\sim 2-\mathrm{dB}$ penalty in terms of received optical power is observed after transmission. For the performance of the N-WDM signal (Fig. 4c, back-to-back; Fig. $4 \mathrm{~d}$, after transmission), we use $33 \%$ overhead for the LDPC coding to adapt to the signal-to-noise ratio of the received signal and achieve error-free performance. The line rate of the $2-\mu \mathrm{m}$-band $4 \times 32$-Gbaud $16-\mathrm{QAM}$ signal is 32 Gbaud $\times 4(16$ QAM modulation $) \times 4(\mathrm{~N}-\mathrm{WDM})=512 \mathrm{Gbit} \mathrm{s}^{-1}$. The net rate is 512 Gbit s $^{-1} \div(1+20 \%) \div(1+33 \%) \div(1+0.8 \%)=318.25$ Gbit s$^{-1}$, excluding the forward error correction (FEC) overheads and the pilot overhead. Since the spectral occupation of the N-WDM signal is only $131.32 \mathrm{GHz}$, the spectral efficiency of $2.4 \mathrm{bit} \mathrm{s}^{-1} \mathrm{~Hz}^{-1}$ is achieved. We have observed a $4.5-\mathrm{dB}$ power penalty for the N-WDM signal after transmission. The penalties observed for the single-channel and N-WDM transmissions mainly come from the losses of the HCF and the receiver-side spectral translator. To the best of our knowledge, this is the first demonstration of a 2- $\mu \mathrm{m}$-band coherent optical communication system with a high capacity and a good spectral efficiency. To improve the conversion efficiencies of the spectral translations, the transmitter-side and receiver-side AlGaAsOI nanowaveguides should be further optimized for the 2- $\mu \mathrm{m}$-band and C-band signals, respectively, to minimize the waveguide loss and coupling loss.

We have proposed and demonstrated a scheme to unlock unconventional wavelength bands for optical coherent communication using a pair of integrated continuous spectral translators based on $\mathrm{CW}$ pumped AlGaAsOI nanowaveguides and high-performance C-band transmitters and receivers. With a cutting-edge short-wavelength TDFA and use of HCF, we have demonstrated the first $2-\mu \mathrm{m}$-band coherent communication system with a high capacity and a good spectral efficiency. The spectral translation scheme could be adapted to other new wavelength bands since the AlGaAsOI nanowaveguides have shown an octave spanning conversion bandwidth. This approach could facilitate the use of new wavelength bands for coherent optical communications by avoiding the need to develop new optical transceivers at those bands. We also believe this scheme has significance in broader applications beyond optical communications, such as in massive photonic integration in TPA-free wavelength bands and in high-resolution spectroscopy for unconventional wavelength bands, through the possibility of coherently transmitting, detecting, and processing signals at the wavelength bands that are currently inaccessible using current signal processing devices. 


\section{Methods}

\section{The AlGaAsOI nanowaveguide}

The fabrication of the AlGaAsOI nanowaveguides starts with a GaAs substrate with a 3- $\mu$ m-thick layer of silicon dioxide and a 350-nm-thick layer of GaAs on top. The aluminium composition ( $\mathrm{x}$ ) for $\mathrm{Al}_{x} \mathrm{Ga}_{1-x} \mathrm{As}$ is $21 \%$. The AlGaAsOI wafer was achieved by wafer bonding and substrate removal ${ }^{40}$. Then, the nanowaveguides and tapers were defined by E-beam lithography followed by a boron trichloride based dry etching process. Both the E-beam process and the dry etching process were optimized to ensure minimal sidewall roughness ${ }^{30}$. The oxide-like E-beam resist hydrogen silsesquioxane was left on top of the nanowaveguide

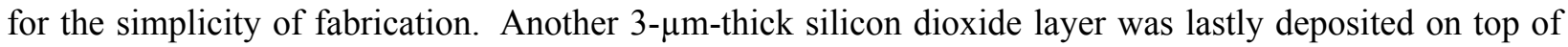
the nanowaveguides by plasma-enhanced chemical vapour deposition to form a cladding. The sample was lastly cleaved at the inverse tapers, which were probed by lensed fibres for light coupling.

Each AlGaAsOI nanowaveguide used in the experiment consists of a straight waveguide and two inverse tapers serving as input and output ports to the waveguide. The core of the nanowaveguide has a high refractive index contrast with the silica cladding from both the top and bottom, leading to strong light confinement ${ }^{29}$. Thus, the required pump power for the spectral translation is significantly reduced. Dispersion engineering is efficiently achieved by adjusting the dimensions (height and width) of the nanowaveguide thanks to the highly confined waveguide mode, enabling a large conversion bandwidth. The high-order phase-matching technique is also used to extend the conversion bandwidth over $1400 \mathrm{~nm}^{31}$. The crosssection dimensions of the straight waveguide are designed to be $910 \times 350 \mathrm{~nm}^{2}$, resulting in a ZDW at 1747.7 nm. A pair of inverse tapers are placed at each side of the straight waveguide, and are optimized for low coupling loss. The nanowaveguide is $5 \mathrm{~mm}$ in length to enable a suitable balance between the conversion bandwidth and efficiency. Although the 910-nm-wide nanowaveguide supports higher-order modes besides the fundamental, the excitation of the higher-order modes is suppressed by the adiabatic design of the inverse taper and fully-straight design of the nanowaveguide.

\section{The 1.74- $\mu \mathrm{m}-\mathrm{band}$ TDFA}

For the $1.74 \mu \mathrm{m}$ pump source, we developed an all-fibre short-wavelength (1650-1800 nm) TDFA using commercially available thulium-doped fibre (TDF) (OFS TmDF200). To enhance the short-wavelength gain of the TDFA a relatively short overall length of TDF $(\sim 1.2 \mathrm{~m})$ was used to reduce the signal reabsorption 
and an additional short-pass spectral filter was incorporated within the amplifier to suppress gain and the build-up of amplified spontaneous emission/spurious lasing at longer wavelengths. The short-pass filter was based on macrobend-induced loss from a 10 m length of dispersion compensating fibre (DCF) (Thorlabs DCF38), wound on a mandrel at an optimized bend diameter of $5.5 \mathrm{~cm}$. This filter was spliced into the middle of the active fibre to increase the available gain at the $1.74-\mu \mathrm{m}$ band $^{34}$. The fibre was core pumped in a bidirectional configuration by an in-house built erbium-doped fibre laser operating at $1560 \mathrm{~nm}$. The amplifier provides a maximum small-signal gain of $>30 \mathrm{~dB}$ at $1750 \mathrm{~nm}$ and supports gain over a more than $200 \mathrm{~nm}$ wide spectral window in the range 1730-1950 nm. In our experiment, the amplifier was seeded by a discrete mode DFB laser diode operating at $1739.74 \mathrm{~nm}$ (Eblana Photonics EP1742-DM-B) and the maximum achievable output power was higher than $500 \mathrm{~mW}$ with an optical signal-to-noise ratio (OSNR) of $>40 \mathrm{~dB}$ (as shown in Fig. 3).

\section{The 1.15-km HCF}

The HCF used in our experiment is a hollow-core photonic bandgap fibre with a 19-cell core structure designed to operate in the $2 \mu \mathrm{m}$ region and was previously fabricated in-house using a conventional stack and draw technique ${ }^{21}$. As shown in the cross-sectional image of the fibre in the inset of Fig. 3, the central hollow core region is surrounded by multiple periodic layers of air holes and glass struts (i.e. micro-structured cladding with a $7 \frac{1}{2}$ ring structure) and most of the light (>99\%) propagates in air rather than silica glass through the photonic bandgap effect. The core diameter is approximately $27 \mu \mathrm{m}$ and the cladding diameter is $\sim 166 \mu \mathrm{m}$ (including the $105 \mu \mathrm{m}$ diameter micro-structured region). The average hole-to-hole spacing is $\sim 6 \mu \mathrm{m}$. The calculated mode field diameter is $\sim 18 \mu \mathrm{m}$ and the effective index of the fundamental mode is $\sim 0.998$. The fibre is $1.15 \mathrm{~km}$ long and shows a wide low loss transmission window centred around $2 \mu \mathrm{m}$, having a minimum loss of $2.8 \mathrm{~dB} \mathrm{~km}^{-1}$ at $1993 \mathrm{~nm}$ and a $3 \mathrm{~dB}$ bandwidth of $86 \mathrm{~nm}$ (1959-2045 nm). Both ends of the HCF were spliced to conventional SSMF and the total link loss was $\sim 9 \mathrm{~dB}$. The link loss was dominated by loss due to the mode field diameter mismatch between the SSMF and HCF. Note that current state-of-the-art HCF now has a much lower attenuation $\left(0.22 \mathrm{~dB} \mathrm{~km}^{-1}\right.$ now reported at wavelengths around $1625 \mathrm{~nm})^{41}$ and much lower interconnection losses can be achieved $(0.15 \mathrm{~dB}$ now reported from SSMF to $\mathrm{HCF})^{42}$, and is emerging as an appealing solution for latency-sensitive optical interconnects. 


\section{Data modulation and digital signal processing}

In the experiment, the C-band $4 \times 32$ Gbaud 16 -QAM signal is generated by four external cavity laser (ECL) and two standard I/Q modulators. The ECLs, each with a linewidth of $10 \mathrm{kHz}$ and output power of $15 \mathrm{dBm}$, are grouped into odd and even channels. The odd and even channels are then independently modulated by the I/Q modulators at 32 Gbaud with different source data. The data comes from a pseudo-random bit sequence with a length of $2^{23}-1$. The data is encoded with LDPC block code from the digital video broadcasting satellite - second generation (DVB-S2) standard and mapped to 16-QAM symbols. Then, the pilot symbols are added. The symbols are digitally shaped into waveforms using a root-raised-cosine filter with 401 taps and 0.01 roll-off. Finally, the waveforms are resampled and loaded to an arbitrary waveform generator with a sampling rate of $64 \mathrm{GSa} \mathrm{s}^{-1}$ for the I/Q signals. The N-WDM signal is launched into the transmitter-side spectral translator with a measured optical power of $21 \mathrm{dBm}$ before the $\mathrm{AlGaAsOI}$ nanowaveguide and then converted to the $2-\mu \mathrm{m}$ band. A 2- $\mu \mathrm{m}$-band TDFA is used as a link amplifier. A fibre-Bragg grating with 4-nm bandwidth with a circulator as an optical filter is used as an optical bandpass fiter (OBPF) to eliminate the excess amplified spontaneous emission noise.

The received 2- $\mu \mathrm{m}$-band N-WDM signal is launched to the receiver-side spectral translator with a measured optical power of $15.7 \mathrm{dBm}$ before the $\mathrm{AlGaAsOI}$ nanowaveguide and then converted back into the C-band. The C-band N-WDM signal is then amplified and filtered by a tuneable OBPF with 3-dB bandwidth of $0.4 \mathrm{~nm}$ to pre-align a target N-WDM channel for performance investigation. The channel-undertest is then launched to the C-band coherent receiver, which consists of a polarization-diversity 90 -degree hybrid, a tuneable local oscillator with $10-\mathrm{kHz}$ linewidth and $16-\mathrm{dBm}$ output power, four pairs of balanced photodetectors, and a digital sampling oscilloscope with $80 \mathrm{GSa} \mathrm{s}^{-1}$ sampling rate and 33-GHz bandwidth. The detected signals are digitally lowpass filtered and resampled to 2 samples per symbol. The signals are then synchronized and equalized using a T/2-spaced pilot-aided radius-directed adaptive equalizer with 51 taps. We use only 51 taps because the chromatic dispersion of the 1.15-km HCF can be neglected. The equalization rectifies inter-symbol interference due to the imperfect frequency response of the transmitter and receiver. A decision-directed phase-locked loop is applied for frequency offset correction and carrier phase recovery. Finally, the signal is LDPC decoded. 


\section{Data Availability}

The data that support the plots within this paper and other findings of this study are available from the corresponding author upon reasonable request.

\section{Code Availability}

The algorithms used for the digital signal processing at the transmitter and the coherent receiver are standard and are outlined in detail in the Methods. MATLAB scripts can be provided by the corresponding authors upon reasonable request.

\section{References}

[1] Essiambre, R.-J., Kramer, G., Winzer, P. J., Foschini, G. J. \& Goebel, B. Capacity limits of optical fiber networks. Journal of Lightwave Technology 28, 662-701 (2010).

[2] Willner, A. E. Optical communications: Innovations and applications abound. JM2A.3 (Optical Society of America, 2021).

[3] Ip, E., Lau, A. P. T., Barros, D. J. F. \& Kahn, J. M. Coherent detection in optical fiber systems. Optics Express 16, 753 (2008).

[4] Wu, X., van den Borne, D., Maki, J. J., Alleston, S. \& Mola, D. D. Interoperable coherent pluggables beyond 400ZR. T2C.4 (Optical Society of America, 2019).

[5] Doerr, C. et al. Single-chip silicon photonics 100-Gb/s coherent transceiver. Th5C.1 (Optical Society of America, 2014).

[6] Winzer, P. J. High-spectral-efficiency optical modulation formats. Journal of Lightwave Technology 30, 3824-3835 (2012).

[7] Winzer, P. J. \& Essiambre, R. Advanced optical modulation formats. Proceedings of the IEEE 94, 952-985 (2006).

[8] Schmogrow, R. et al. Nyquist frequency division multiplexing for optical communications. CTh1H.2 (Optical Society of America, 2012). 
[9] Hillerkuss, D. et al. Single-laser 32.5 Tbit/s Nyquist WDM transmission. IEEE/OSA Journal of Optical Communications and Networking 4, 715-723 (2012).

[10] Lowery, A. J. \& Armstrong, J. Orthogonal-frequency-division multiplexing for dispersion compensation of long-haul optical systems. Opt. Express 14, 2079-2084 (2006).

[11] Ip, E. M. \& Kahn, J. M. Fiber impairment compensation using coherent detection and digital signal processing. Journal of Lightwave Technology 28, 502-519 (2010).

[12] Ip, E. \& Kahn, J. M. Compensation of dispersion and nonlinear impairments using digital backpropagation. J. Lightwave Technol. 26, 3416-3425 (2008).

[13] Buchali, F. et al. $52.1 \mathrm{~Tb} / \mathrm{s}$ C-band DCI transmission over DCI distances at $1.49 \mathrm{~Tb} / \mathrm{s} / \lambda$. $1-4$ (2020).

[14] Ionescu, M. et al. $91 \mathrm{~nm} \mathrm{C+L} \mathrm{hybrid} \mathrm{distributed} \mathrm{raman-erbium-doped} \mathrm{fibre} \mathrm{amplifier} \mathrm{for} \mathrm{high} \mathrm{capacity}$ subsea transmission. 1-3 (2018).

[15] Renaudier, J. et al. First 100-nm continuous-band WDM transmission system with 115Tb/s transport over 100km using novel ultra-wideband semiconductor optical amplifiers. 1-3 (2017).

[16] Essiambre, R.-J. \& Tkach, R. W. Capacity trends and limits of optical communication networks. Proceedings of the IEEE 100, 1035-1055 (2012).

[17] Ellis, A. D., Zhao, J. \& Cotter, D. Approaching the non-linear shannon limit. Journal of Lightwave Technology 28, 423-433 (2010).

[18] Gunning, F. \& Corbett, B. Time to open the 2- $\mu$ m window? Opt. Photon. News 30, 42-47 (2019).

[19] Suibhne, N. M. et al. WDM transmission at $2 \mu \mathrm{m}$ over low-loss hollow core photonic bandgap fiber. OW1I.6 (Optical Society of America, 2013).

[20] Liu, Z. et al. High-capacity directly modulated optical transmitter for $2-\mu \mathrm{m}$ spectral region. Journal of Lightwave Technology 33, 1373-1379 (2015).

[21] Zhang, H. et al. $100 \mathrm{Gbit} / \mathrm{s}$ WDM transmission at $2 \mu \mathrm{m}$ : transmission studies in both low-loss hollow core photonic bandgap fiber and solid core fiber. Optics Express 23, 4946-4951 (2015). 
[22] Petrovich, M. N. et al. Demonstration of amplified data transmission at $2 \mu \mathrm{m}$ in a low-loss wide bandwidth hollow core photonic bandgap fiber. Optics Express 21, 28559 (2013).

[23] Koizumi, K., Yoshida, M., Hirooka, T. \& Nakazawa, M. 160 Gbit/s-300 km single-channel transmission in the $1.1 \mu \mathrm{m}$ band with a precise GVD and slope compensation. Opt. Express 21, 4303-4310 (2013).

[24] Koizumi, K., Yoshida, M., Hirooka, T. \& Nakazawa, M. A single-channel 1.28 Tbit/s-58 km transmission in the $1.1 \mu \mathrm{m}$ band with wideband GVD and slope compensation. Optics Express 21, 29055 (2013).

[25] Kurokawa, K. et al. High capacity WDM transmission in $1.0 \mu \mathrm{m}$ band over low loss PCF using supercontinuum source. OMH5 (Optical Society of America, 2008).

[26] Liu, X. et al. Bridging the mid-infrared-to-telecom gap with silicon nanophotonic spectral translation. Nature Photonics 6, 667-671 (2012).

[27] Ophir, N. et al. First demonstration of a 10-gb/s RZ end-to-end four-wave-mixing based link at 1884 nm using silicon nanowaveguides. IEEE Photonics Technology Letters 24, 276-278 (2012).

[28] Lu, X. et al. Efficient telecom-to-visible spectral translation through ultralow power nonlinear nanophotonics. Nature Photonics 13, 593-601 (2019).

[29] $\mathrm{Pu}, \mathrm{M}$. et al. Ultra-efficient and broadband nonlinear AlGaAs-on-insulator chip for low-power optical signal processing. Laser \& Photonics Reviews 10.1002/lpor.201800111 (2018).

[30] Pu, M., Ottaviano, L., Semenova, E. \& Yvind, K. Efficient frequency comb generation in AlGaAs-oninsulator. Optica 3, 823-826 (2016).

[31] Liu, Y. et al. High-order phase-matching enabled octave-bandwidth four-wave mixing in AlGaAs-oninsulator waveguides. JTu2A.83 (OSA, 2019).

[32] Kong, D. et al. 744-nm wavelength conversion of PAM-4 signal using an AlGaAsOI nanowaveguide. Optics Letters 45, 889-892 (2020).

[33] Shen, W. et al. Low-latency and high-speed hollow-core fiber optical interconnection at 2-micron waveband. J. Lightwave Technol. 38, 3874-3882 (2020). 
[34] Li, Z. et al. Exploiting the short wavelength gain of silica-based thulium-doped fiber amplifiers. Optics Letters 41, 2197-2200 (2016).

[35] Ip, E. \& Kahn, J. M. Feedforward carrier recovery for coherent optical communications. Journal of Lightwave Technology 25, 2675-2692 (2007).

[36] Colavolpe, G., Barbieri, A. \& Caire, G. Algorithms for iterative decoding in the presence of strong phase noise. IEEE Journal on Selected Areas in Communications 23, 1748-1757 (2005).

[37] Cheng, H. et al. Low overhead slipless carrier phase estimation scheme. Optics Express 22, 2074020747 (2014).

[38] Zhang, J. et al. Single-frequency 1.7- $\mu \mathrm{m}$ Tm-doped fiber laser with optical bistability of both power and longitudinal mode behavior. Opt. Express 29, 21409-21417 (2021).

[39] Millar, D. S. et al. Design of a $1 \mathrm{~Tb} / \mathrm{s}$ superchannel coherent receiver. Journal of Lightwave Technology 34, 1453-1463 (2016).

[40] Ottaviano, L., Pu, M., Semenova, E. \& Yvind, K. Low-loss high-confinement waveguides and microring resonators in AlGaAs-on-insulator. Optics Letters 41, 3996-3999 (2016).

[41] Sakr, H. et al. Hollow core NANFs with five nested tubes and record low loss at 850, 1060, 1300 and 1625nm. F3A.4 (Optical Society of America, 2021).

[42] Suslov, D. et al. Low loss and high performance interconnection between standard single-mode fiber and antiresonant hollow-core fiber. Scientific Reports 11, 8799 (2021).

[43] Tkach, R., Chraplyvy, A., Forghieri, F., Gnauck, A. \& Derosier, R. Four-photon mixing and high-speed WDM systems. Journal of Lightwave Technology 13, 841-849 (1995).

[44] Li, Z. et al. Diode-pumped wideband thulium-doped fiber amplifiers for optical communications in the 1800 - $2050 \mathrm{~nm}$ window. Opt. Express 21, 26450-26455 (2013).

[45] Li, Z. et al. Thulium-doped fiber amplifier for optical communications at $2 \mu \mathrm{m}$. Optics Express 21, 9289-9297 (2013).

[46] Roberts, P. J. et al. Ultimate low loss of hollow-core photonic crystal fibres. Opt. Express 13, 236-244 (2005). 
[47] Chigusa, Y. Ultra-low-loss $(0.1484 \mathrm{db} / \mathrm{km})$ pure silica core fibre and extension of transmission distance. Electronics Letters 38, 1168-1169(1) (2002).

[48] Sakr, H. et al. Ultrawide bandwidth hollow core fiber for interband short reach data transmission. 1-3 (2019).

[49] Slavík, R. et al. $1.45 \mathrm{Tbit} / \mathrm{s}$, low latency data transmission through a 19-cell hollow core photonic band gap fibre. Mo.2.F.2 (Optical Society of America, 2012).

[50] Liu, Z. et al. Nonlinearity-free coherent transmission in hollow-core antiresonant fiber. Journal of Lightwave Technology 37, 909-916 (2019).

[51] Liu, X., Osgood, R. M., Vlasov, Y. A. \& Green, W. M. J. Mid-infrared optical parametric amplifier using silicon nanophotonic waveguides. Nature Photonics 4, 557-560 (2010).

[52] Zlatanovic, S. et al. Mid-infrared wavelength conversion in silicon waveguides using ultracompact telecom-band-derived pump source. Nature Photonics 4, 561-564 (2010).

[53] Galili, M. et al. Generation and detection of 2.56 Tbit/s OTDM data using DPSK and polarisation multiplexing. OThV2 (OSA, 2010).

\section{Acknowledgements}

H.H. acknowledges the research grant (15401) of the Young Investigator Program (2MAC) from the VILLUM FONDEN. L.K.O acknowledges funding from the Danish National Research Foundation (DNRF) through the Research Centre of Excellence, Silicon Photonics for Optical Communications (SPOC) (ref. DNRF123). D.J.R acknowledges the UK Engineering and Physical Sciences Research Council (EPSRC) through the “Airguide Photonics" Programme Grant (EP/P030181/1). N.V.W acknowledges the Royal Society (Univer-

sity Research Fellowship). The authors gratefully acknowledge Francesco Poletti for his contribution to the design and fabrication of the 19-cell HCF. 
The CE of degenerate FWM can be determined by CE $=\gamma^{2} P_{\mathrm{p}}^{2} L_{\mathrm{eff}}^{2} \eta$, where $\gamma$ is the nonlinear coefficient, and $P_{\mathrm{p}}$ is the optical power of the pump. $L_{\text {eff }}$ is the effective length of the nonlinear waveguide and $L_{\text {eff }}=$ $\left(1-e^{-\alpha L}\right)$, where $\alpha$ is the loss per unit length and $L$ is the length of the waveguide. The normalised CE is defined as ${ }^{43}$ :

$$
\eta=\frac{\alpha^{2}}{\alpha^{2}+\Delta \beta^{2}}\left(1+\frac{4 e^{-\alpha L} \sin ^{2}(\Delta \beta L / 2)}{\left(1-e^{-\alpha L}\right)^{2}}\right)
$$

where $\Delta \beta$ is the phase mismatch per unit length of the waveguide. The phase mismatch is a sum of linear and power-dependent nonlinear terms:

$$
\Delta \beta=\Delta \beta_{\text {linear }}+2 \gamma P_{\mathrm{p}}
$$

where $\Delta \beta_{\text {linear }}=2 \beta_{\mathrm{p}}-\beta_{\mathrm{s}}-\beta_{\mathrm{i}}$, and $\beta_{\mathrm{p}}, \beta_{\mathrm{s}}, \beta_{\mathrm{i}}$ are the propagation constant of the pump, signal, and idler, respectively. Here we assume that the optical power of the signal and the idler is significantly smaller than the pump power. Consequently, the nonlinear phase mismatch originates from self-phase modulation of the pump. The overall phase mismatch term should be minimized for a large CE. If the pump is in the small power region the contribution of nonlinear phase mismatch can be ignored. The linear phase mismatch ban be expanded at the pump frequency and simplified to

$$
\Delta \beta_{\text {linear }}=\beta_{2} \Delta \omega^{2}+\frac{1}{12} \beta_{4} \Delta \omega^{4}
$$


where the first and second terms come from the second and fourth-order dispersion. To minimize the phase mismatch, i.e., $\Delta \beta_{\text {linear }}=0$, we have

$$
\Delta \omega= \pm \sqrt{-12\left(\beta_{2} / \beta_{4}\right)}
$$

This means that two phase matching points exist in addition to the one where the signal and the pump have the same frequency (i.e., $\Delta \omega=0$ ). The conversion bands associated with these phase matching points are defined as high-order phase matching bands. As these phase matching points are a function of the pump frequency, a large continuous conversion band can be achieved by detuning the pump from the ZDW and exploiting the high-order phase matching bands.

\section{Advantages of the 2- $\mu \mathrm{m}$ band}

The $2-\mu \mathrm{m}$ band is currently a leading contender among all the new wavelength bands due to the combination of several emerging technologies. Firstly, the TDFA offers more than 240-nm bandwidth in the 2- $\mu \mathrm{m}$ band with high gain and low noise figure ${ }^{44,45}$. Secondly, HCF, e.g., using photonic bandgap or anti-resonant effects, enables data transmission in the $2-\mu \mathrm{m}$ band. HCF could potentially achieve a loss of the order of 0.1 $\mathrm{dB} \mathrm{km}^{-146}$, surpassing the best conventional silica fibres ${ }^{47}$. Photonic bandgap fibres have been made with $>150-\mathrm{nm}$ bandwidth in the $2-\mu \mathrm{m}$ band $^{22}$ and antiresonant fibres with $700-\mathrm{nm}$ bandwidth covering both the conventional $\mathrm{C}$ band and the $2-\mu \mathrm{m}$ band $^{48}$. HCF also features low latency transmission at $>99.7 \%$ of the speed of light ${ }^{49}$, and ultralow nonlinearity ${ }^{50}$. Just as low-loss SSMF and the EDFA have boosted the optical fibre telecom industry, HCF and the TDFA could ultimately spawn novel $2-\mu \mathrm{m}$-band optical communication systems, allowing signals to be relayed over long distances with low cost and low energy consumption. In addition, the $2-\mu \mathrm{m}$ band is attractive for silicon photonics due to the reduced two-photon absorption ${ }^{51,52}$, allowing advanced on-chip functionalities.

\section{Measured spectral flatness of the translation}

We have measured the flatness of the translation band with CW signal sweeping from $1500 \mathrm{~nm}$ to $1580 \mathrm{~nm}$ with an interval of $0.5 \mathrm{~nm}$. The results are shown in Fig. S1 with scattered points, along with a comparison to the simulation results. Note that, in this measurement, the pump originates from a DFB laser working at $1739.18 \mathrm{~nm}$. The solid and dash-dotted curves show simulation results for the translation band when the 


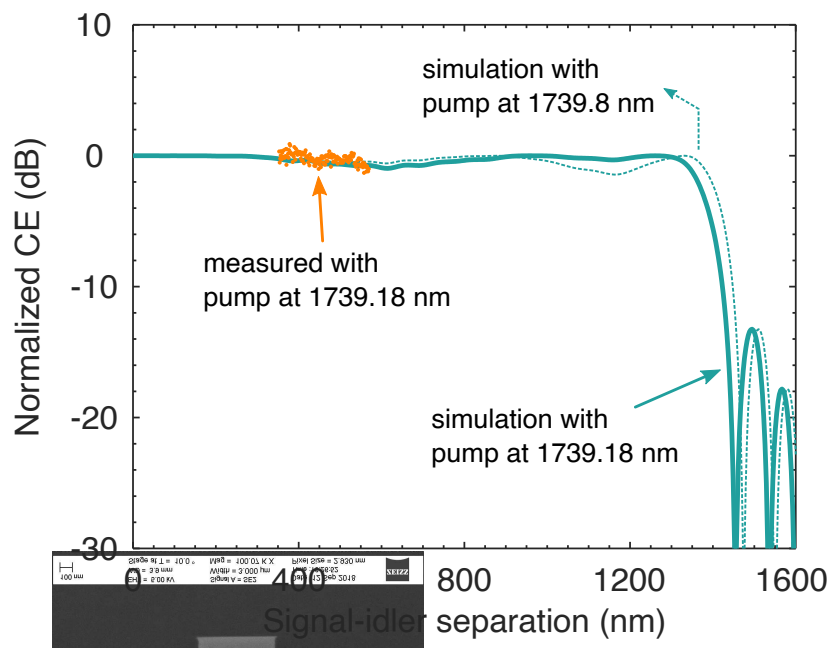

Fig. S1 | Measured flatness of the translation band. The measurement is done with a CW signal sweeping from $1500 \mathrm{~nm}$ to $1580 \mathrm{~nm}$, while the pump is a CW from a DFB laser at $1739.18 \mathrm{~nm}$. The continuous curve shows the simulation results with a pump wavelength of $1739.18 \mathrm{~nm}$. The dashed curve shows the simulation results with the pump at the optimum wavelength of $1739.8 \mathrm{~nm}$.

pump is tuned to $1739.18 \mathrm{~nm}$ and the optimum wavelength of $1739.8 \mathrm{~nm}$, respectively. It can be seen that an offset from the optimum pump wavelength would result in a slightly decreased conversion bandwidth, but the conversion bandwidth is still sufficient for the spectral translation from the $\mathrm{C}$ band to the $2-\mu \mathrm{m}$ band and vice versa. In the $2-\mu \mathrm{m}$-band transmission experiment, the pump laser is thermally tuned to $1739.74 \mathrm{~nm}$, which is very close to the optimum pump wavelength. Nevertheless, the measured results of the flatness of the translation band match well with the simulation and a maximum fluctuation of the conversion efficiency of $<2 \mathrm{~dB}$ is achieved.

\section{Spectral translation of an optical frequency comb}

In addition to the transmission experiment, we have also performed spectral translation of an optical frequency comb (OFC) from the $\mathrm{C}$ band to the $2-\mu \mathrm{m}$ band to explore the potential of our spectral translators. The OFC originates from a seed 1.5-ps Gaussian pulse train out of a mode-locked laser with a centre wavelength of $1543.5 \mathrm{~nm}$, a repetition rate of $10 \mathrm{GHz}$, and a $20-\mathrm{dB}$ spectral width of $6.4 \mathrm{~nm}$. The seed OFC from the laser is spectrally broadened based on self-phase modulation in a piece of 400-m dispersion-flattened highly nonlinear fibre (DF-HNLF) $\left(\gamma=10.5 \mathrm{~W}^{-1} \mathrm{~km}^{-1}, \beta_{2}=0.567 \mathrm{ps}^{2} / \mathrm{km}, \beta_{3}=0.008 \mathrm{ps}^{3} / \mathrm{km}\right)^{53}$ with a launch power of $26.5 \mathrm{dBm}$, resulting in a broadened OFC with a 10-dB bandwidth of $40 \mathrm{~nm}$ spanning from 


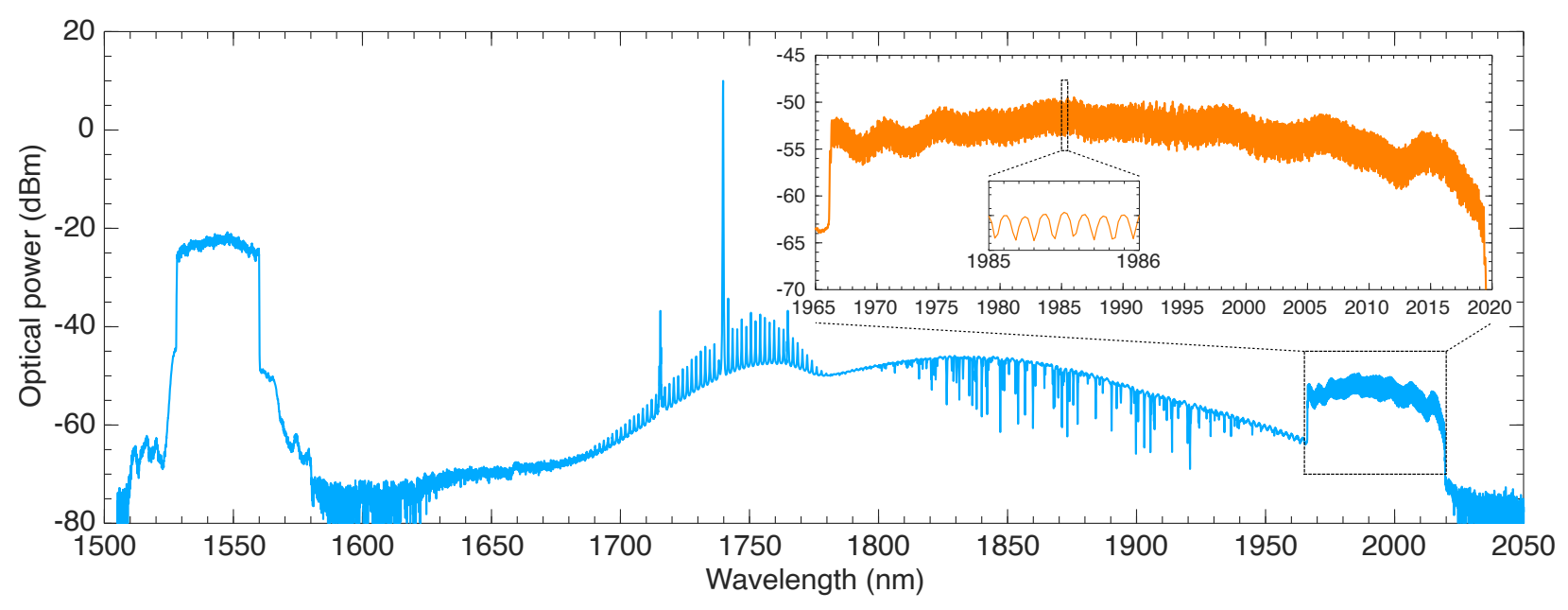

Fig. S2 | Spectral translation of a 10-GHz spaced optical frequency comb covering most of the telecom $\mathrm{C}$ band $(1528.0 \mathrm{~nm}$ to $1560.0 \mathrm{~nm})$ to the $2-\mu \mathrm{m}$ band. The result demonstrates that the AlGaAsOI nanowaveguide based spectral translator has a flat conversion band and is capable of the spectral translation of a wideband multi-channel signal.

$1524.0 \mathrm{~nm}$ to $1563.9 \mathrm{~nm}$. Then, the OFC is spectrally shaped by a wavelength-selective switch to a flat-top spectrum spanning from $1528.0 \mathrm{~nm}$ to $1560.0 \mathrm{~nm}$. After amplification, the OFC is aligned in polarization with the pump and finally launched into the spectral translator to be converted to the $2-\mu \mathrm{m}$ band. Figure S2 gives the output spectra from the spectral translator, with the zoomed-in spectra showing the converted $2-\mu \mathrm{m}$-band OFC. The shape of the OFC is well preserved after the spectral translator, confirming a flat conversion bandwidth across the telecom $\mathrm{C}$ band. The large conversion bandwidth shown in the simulation results and experimental measurements demonstrates that the spectral translator could be a very promising solution to bridge many wavelength bands.

\section{Latency measurement for the HCF}

Despite the wide transmission band for the $2-\mu \mathrm{m}$ signal, the HCF has an overwhelming advantage in terms of latency (pulse propagation delay). We have measured the latency of the $\sim 1.15-\mathrm{km} \mathrm{HCF}$ for the $2-\mu \mathrm{m}$-band signal, with a comparison of the latency of a C-band signal transmitting through a $1.15-\mathrm{km} \mathrm{SSMF}$. We use a pulse train generated from an arbitrary waveform generator with a repetition rate of $100-\mathrm{kHz}$ and a duty cycle of $1 \%$. The pulse train is modulated into the optical domain by Mach-Zehnder modulators (Fujitsu FTM7938EZ for C band and EOSpace AZ-0S5-20-PFA-PFA-2000-LV for 2- $\mu \mathrm{m}$ band). A photodiode (Dis- 

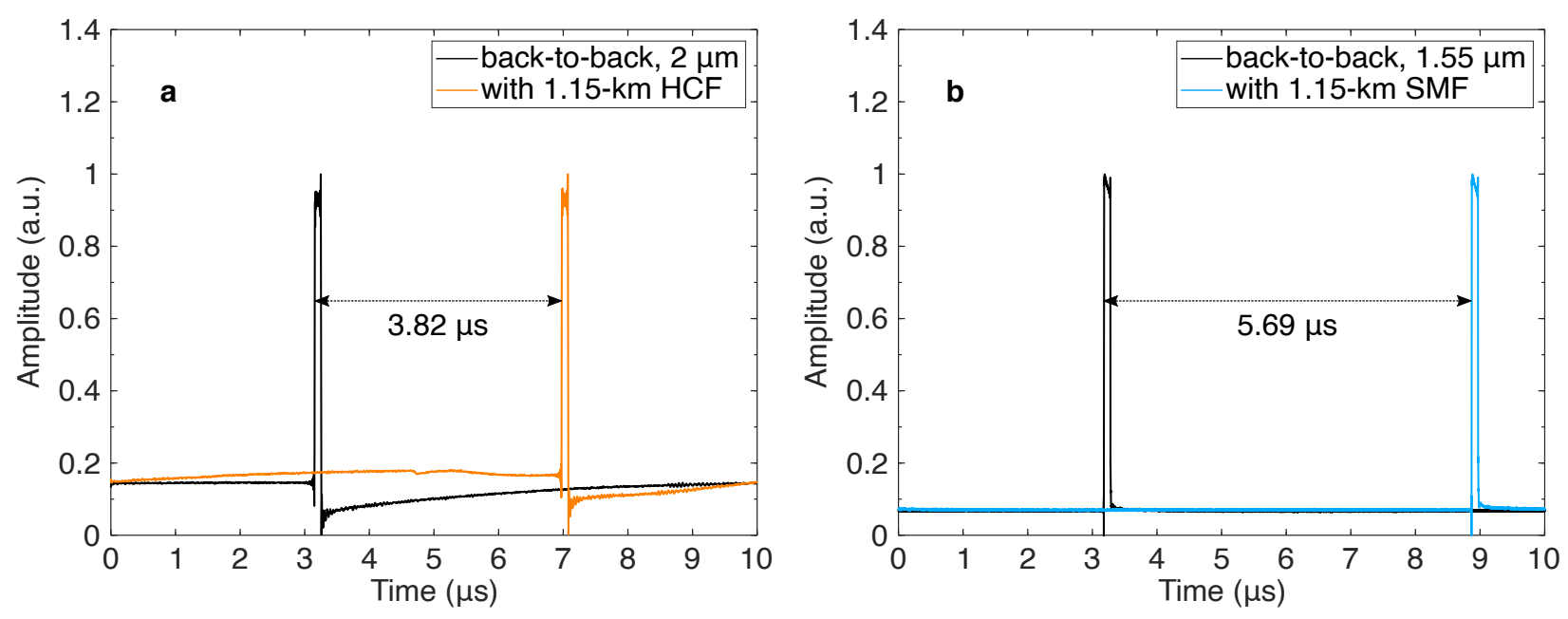

Fig. S3 | Measured pulse propagation delays. a, Pulse propagation delay of the $2-\mu \mathrm{m}$ signal through the 1.15-km HCF. b, Pulse propagation delay of the C-band signal through the 1.15-km SSMF.

covery Semiconductor DSC2-50S) with a 3-dB bandwidth of $12 \mathrm{GHz}$ and a spectral wavelength range from $1200 \mathrm{~nm}$ to $2200 \mathrm{~nm}$ is used to detect the pulse trains before and after transmission. The measured results for the 2- $\mu \mathrm{m}$-band and C-band pulse propagation delays are shown in Fig. S3a and Fig. S3b, respectively. Despite some inaccuracy in the exact length of the HCF and SSMF, the pulse propagation delay is reduced from $5.69 \mu$ s to $3.82 \mu$ s by shifting from the C band with SSMF to the $2-\mu \mathrm{m}$ band in the HCF, showing a latency reduction of $33 \%$, as anticipated. 


\section{Supplementary Files}

This is a list of supplementary files associated with this preprint. Click to download.

- SupplementaryInfo.pdf 